\title{
LWIR Hyperspectral Change Detection for Target Acquisition and Situation Awareness in Urban Areas
}

\author{
Rob J. Dekker ${ }^{* a}$, Piet B.W. Schwering ${ }^{\mathrm{a}}$, Koen W. Benoist ${ }^{\mathrm{a}}$, Stefano Pignatti ${ }^{\mathrm{b}}$, Federico Santini ${ }^{\mathrm{b}}$, \\ Ola Friman ${ }^{\mathrm{c}}$ \\ ${ }^{\mathrm{a}}$ TNO, PO Box 96864, $2509 \mathrm{JG}$ The Hague, The Netherlands; ${ }^{\mathrm{b}} \mathrm{CNR}$ IMAA, Zona Industriale, 85050 \\ Tito Scalo (PZ), Italy; ${ }^{\mathrm{C}} \mathrm{FOI}, \mathrm{PO}$ Box 1165, 58111 Linköping, Sweden
}

\begin{abstract}
This paper studies change detection of LWIR (Long Wave Infrared) hyperspectral imagery. Goal is to improve target acquisition and situation awareness in urban areas with respect to conventional techniques. Hyperspectral and conventional broadband high-spatial-resolution data were collected during the DUCAS trials in Zeebrugge, Belgium, in June 2011. LWIR data were acquired using the ITRES Thermal Airborne Spectrographic Imager TASI-600 that operates in the spectral range of 8.0-11.5 $\mu \mathrm{m}$ (32 band configuration). Broadband data were acquired using two aeroplanemounted FLIR SC7000 MWIR cameras. Acquisition of the images was around noon. To limit the number of false alarms due to atmospheric changes, the time interval between the images is less than 2 hours. Local co-registration adjustment was applied to compensate for misregistration errors in the order of a few pixels. The targets in the data that will be analysed in this paper are different kinds of vehicles. Change detection algorithms that were applied and evaluated are Euclidean distance, Mahalanobis distance, Chronochrome (CC), Covariance Equalisation (CE), and Hyperbolic Anomalous Change Detection (HACD). Based on Receiver Operating Characteristics (ROC) we conclude that LWIR hyperspectral has an advantage over MWIR broadband change detection. The best hyperspectral detector is HACD because it is most robust to noise. MWIR high spatial-resolution broadband results show that it helps to apply a false alarm reduction strategy based on spatial processing.
\end{abstract}

Keywords: LWIR, MWIR, hyperspectral, broadband, change detection

\section{INTRODUCTION}

Within the DUCAS project ${ }^{1}$ seven European countries work together on improved target acquisition and situation awareness in urban areas. One of the goals is to combine hyperspectral and high spatial-resolution active and passive sensors, and to compare those with conventional techniques. To facilitate joint research and development a data acquisition campaign was organised at Zeebrugge, Belgium, in June 2011. Among the acquiring sensors was the ITRES Thermal Airborne Spectrographic Imager TASI- $600^{2}$, a LWIR hyperspectral imager that was operated by CNR IMAA, Italy. The conventional sensors to compare with were two aeroplane-mounted FLIR SC7000 MWIR ${ }^{3}$ broadband highspatial-resolution cameras, with an Indium Antimonide detector, that were operated by FOI, Sweden. This paper shows the analysis and performance of those data in combination with vehicle change detection. In our opinion change detection is the most effective strategy to detect relocated targets in urban environments if no a-priori spectral information is available. Anomaly detection in urban environments generally results in a significant number of false alarms. An alternative strategy based on two successive images is spectral matching ${ }^{4}$, assuming that the potential targets of interest in the first image are known to sample their spectra. Change detection algorithms that are studied in this paper are Euclidean distance, Mahalanobis distance, Chronochrome (CC), Covariance Equalisation (CE), and Hyperbolic Anomalous Change Detection (HACD). Evaluation is done by computing Receiver Operating Characteristics (ROC) based on in-situ collected ground truth.

*rob.dekker@tno.nl; phone +31 8886640 76; http://www.tno.nl/

Algorithms and Technologies for Multispectral, Hyperspectral, and Ultraspectral Imagery XIX, edited by Sylvia S. Shen, Paul E. Lewis, Proc. of SPIE Vol. 8743, 874306 · (C) 2013 SPIE CCC code: $0277-786 X / 13 / \$ 18 \cdot$ doi: $10.1117 / 12.2015761$ 


\section{HYPERSPECTRAL AND BROADBAND DATA}

\subsection{LWIR hyperspectral data and pre-processing}

Collecting data with the TASI-600, chosen was for a 32 band configuration with $109.5 \mathrm{~nm}$ spectral width (FWHM). Spectral range is $8.0-11.5 \mu \mathrm{m}$. Spatial resolution of the images is $0.60 \mathrm{~m}$. Pre-processing of the TASI-600 data includes spectral calibration, radiometric calibration (spectral radiance, $\mu \mathrm{W} \mathrm{cm} \mathrm{sr}^{-1} \mathrm{~nm}^{-1}$ ), blinking pixel removal, and georeferencing ${ }^{5}$. In doing this CNR IMAA used a combination of ITRES software and their own algorithms, the latter mainly for blinking pixel removal. Images that are selected to analyse change detection performance were acquired on 27 June 2011, the first image at UTC 11:53 and the second image at UTC 13:11 (Fig. 1). Area of interest is $230 \times 140 \mathrm{~m}$. Weather conditions were clear sky and sunny. Surface wetness during acquisition was below $15 \%$. Air temperature at UTC 12:00 was $30^{\circ} \mathrm{C}$. Planning was also to apply atmospheric correction, and if possible Temperature-Emissivity Separation (TES), but those are delayed. Since the acquisitions are 1 hour and 18 minutes apart, atmosphere and temperature are assumed to be nearly similar. Georeferencing RMSE of the selected acquisitions is $1.8 \mathrm{~m} \mathrm{(3} \mathrm{pixels),} \mathrm{but}$ co-registration seemed to be significantly better in the areas where relocated vehicles were present. However, additional co-registration adjustment is necessary to reduce the number of false alarms. Ground truth was collected from the selected TASI images and high spatial-resolution $(10 \mathrm{~cm}$ ) visual images acquired during the same period of time (Fig. 2). Eighteen relocated vehicles were identified.
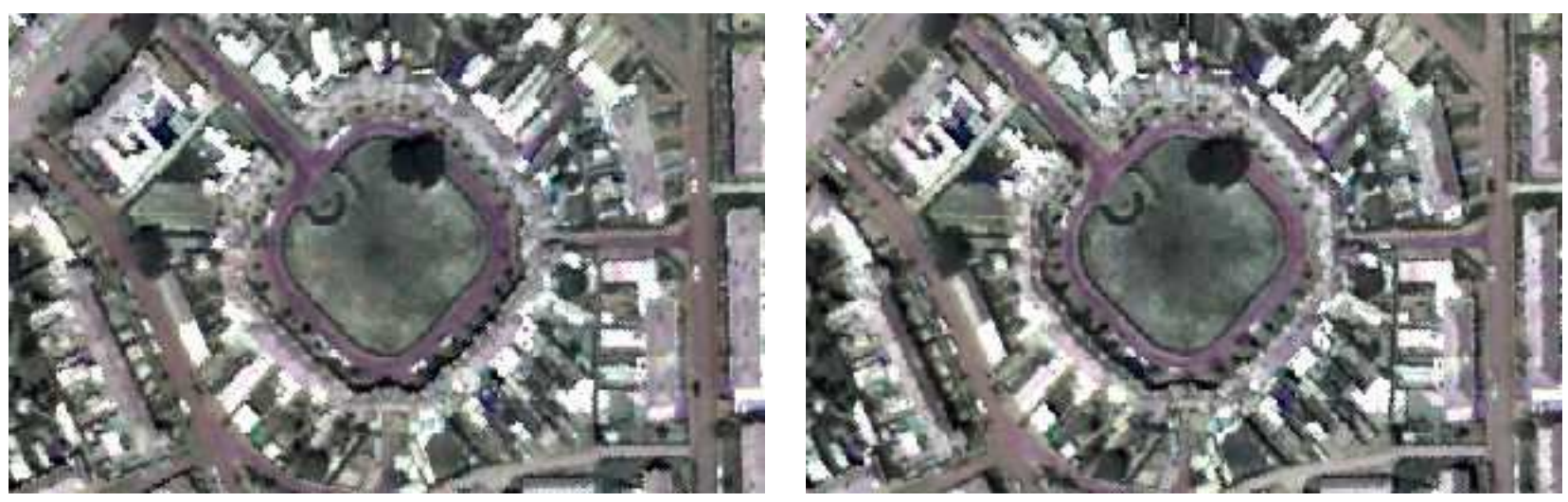

Figure 1. TASI images and area of interest of 27 June 2011 UTC 11:53 (left) and UTC 13:11 (right). Red = 11.45 $\mu \mathrm{m}$; Green $=9.70 \mu \mathrm{m} ;$ Blue $=8.05 \mu \mathrm{m}$. Spatial resolution is $0.60 \mathrm{~m}$.
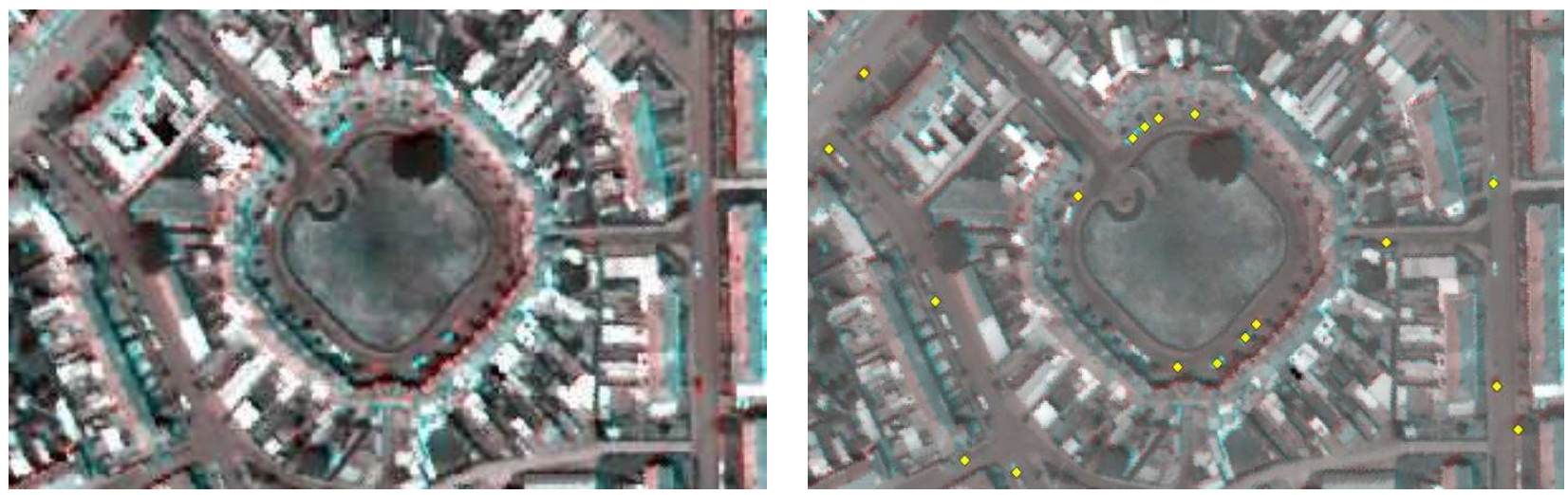

Figure 2. TASI colour composite (left) and ground truth (right). Red $=9.70 \mu \mathrm{m}$, UTC 13:11; Cyan = 9.70 $\mu \mathrm{m}$, UTC 11:53. 


\subsection{MWIR broadband data and pre-processing}

The two FLIR SC7000 MWIR cameras that were used for acquiring conventional broadband infrared data were operating at $3.9 \mu \mathrm{m}$ and $4.8 \mu \mathrm{m}$, both with a spectral width of $600 \mathrm{~nm}$ (FWHM). Spatial resolution of the images is 0.25 $\mathrm{m}$. Pre-processing of the MWIR data includes radiometric calibration (radiance, $\mathrm{W} \mathrm{m}^{-2} \mathrm{sr}^{-1}$ ), mosaicking, and georeferencing. Images that are selected to analyse change detection performance on 27 June 2011, were acquired at UTC 12:17 and at UTC 13:38 (Fig. 3). Additional co-registration was applied by using a first order polynomial in combination with nearest-neighbour resampling. Result was that the residual co-registration error was up to a few pixels in certain regions, but (as in the TASI images) significantly better in regions where relocated vehicles were situated. Here too additional co-registration adjustment is necessary to reduce the number of false alarms. Ground truth was collected directly from the selected FLIR images (Fig. 4). Twenty-two relocated vehicles were identified.
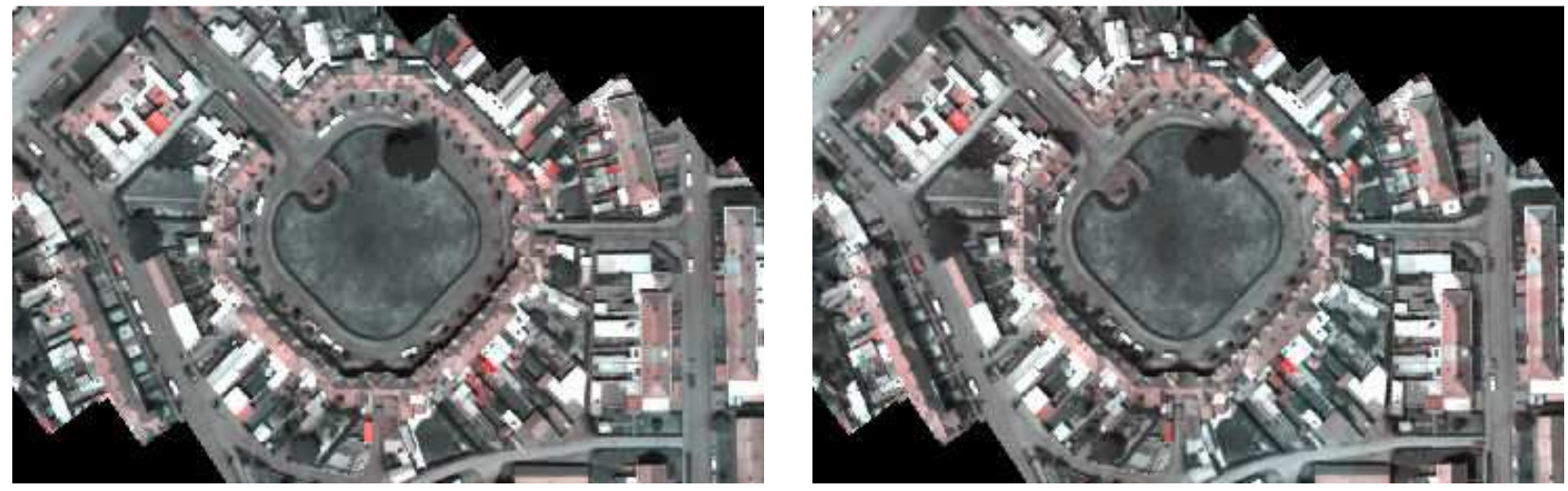

Figure 3. FLIR MWIR images and area of interest of 27 June 2011 UTC 12:17 (left) and UTC 13:38 (right). Red = $3.9 \mu \mathrm{m}$; Cyan $=4.8 \mu \mathrm{m}$. Spatial resolution is $0.25 \mathrm{~m}$.
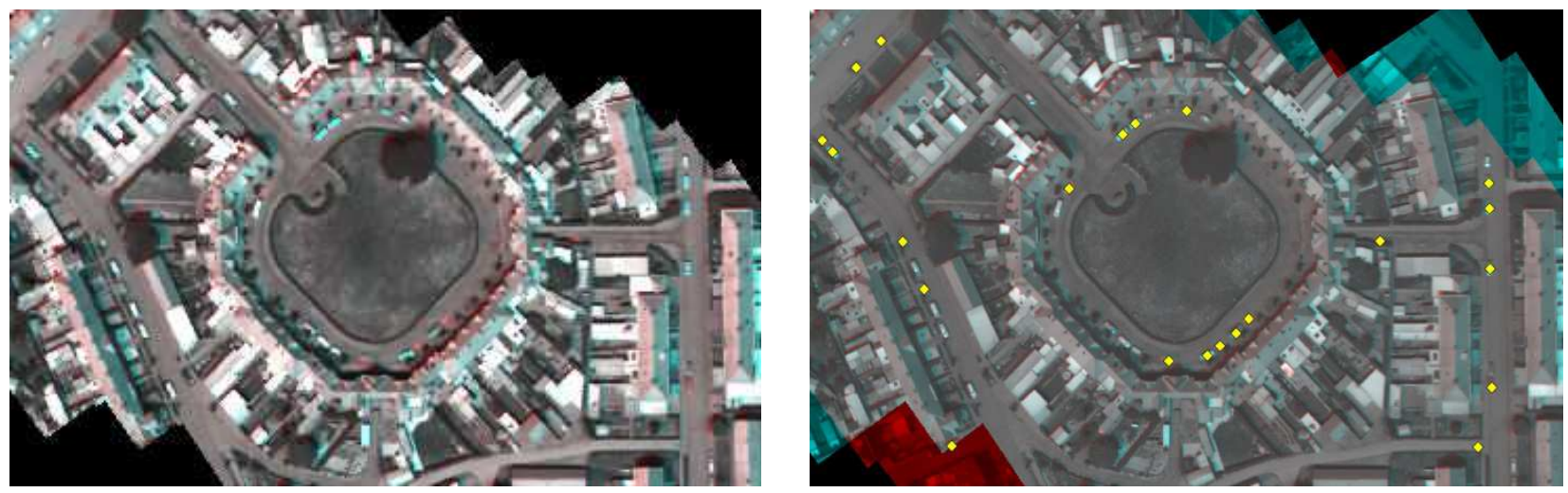

Figure 4. FLIR MWIR colour composite (left) and ground truth (right). Red = $3.9 \mu \mathrm{m}$, UTC 13:38; Cyan $=3.9 \mu \mathrm{m}$, UTC $12: 17$.

\section{METHODOLOGY}

The evaluation of LWIR hyperspectral imagery and MWIR broadband imagery in the context of several change detection strategies is done at object level, after spatial clustering of target pixels. We will show that this has implications to the computation of ROC curves. 


\subsection{Change detection algorithms}

Basically, change detection boils down to the difference between two successive zero-mean spectral measurement vectors $\mathbf{x}$ and $\mathbf{y}$ of the same geospatial position or pixel. The result is referred to as the residual vector:

$$
\boldsymbol{\delta}=\mathbf{x}-\mathbf{y}
$$

The Euclidean distance is simply the length of that vector:

$$
d(\mathbf{x}, \mathbf{y})=\|\boldsymbol{\delta}\|
$$

The Mahalanobis distance is relative to the covariance matrix $\Gamma$ of the residual vector:

$$
d(\mathbf{x}, \mathbf{y})=\boldsymbol{\delta}^{T} \boldsymbol{\Gamma}^{-1} \boldsymbol{\delta}
$$

To compensate for calibration, color balance, or diurnal differences, several prediction strategies can be followed. Both that will be discussed are based on the following matrices:

$$
\begin{array}{r}
\mathbf{X}=\left\langle\mathbf{x} \mathbf{x}^{T}\right\rangle \\
\mathbf{Y}=\left\langle\mathbf{y y}^{T}\right\rangle \\
\mathbf{C}=\left\langle\mathbf{x y} \mathbf{y}^{T}\right\rangle
\end{array}
$$

The first strategy is Chronochrome (CC) processing 6 , 7, 8, 9, 10,11:

$$
\boldsymbol{\delta}_{C C}=\mathbf{x}-\mathbf{C Y} \mathbf{Y}^{-1} \mathbf{y}
$$

Here the second part is the prediction of $\mathbf{x}$ at the time of $\mathbf{y}$. The distance can be computed by using Eq. (3) and replace the residual vector with Eq. (7). The second strategy is Covariance Equalisation (CE) $)^{8,9,10,11}$ :

$$
\boldsymbol{\delta}_{C E}=\mathbf{x}-\mathbf{X}^{\frac{1}{2}} \mathbf{Y}^{-\frac{1}{2}} \mathbf{y}
$$

Comparative studies have shown that $\mathrm{CC}$ and $\mathrm{CE}$ are surpassed in performance by applying segmented linear prediction ${ }^{10}$ or distribution-based detection ${ }^{911}$. The latter, also referred to as Hyperbolic Anomalous Change Detection (HACD) is described by:

$$
a(\mathbf{x}, \mathbf{y})=\left[\begin{array}{ll}
\mathbf{x}^{T} & \mathbf{y}^{T}
\end{array}\right]\left(\left[\begin{array}{cc}
\mathbf{X} & \mathbf{C}^{T} \\
\mathbf{C} & \mathbf{Y}
\end{array}\right]^{-1}-\left[\begin{array}{cc}
\mathbf{X} & 0 \\
0 & \mathbf{Y}
\end{array}\right]^{-1}\right)\left[\begin{array}{l}
\mathbf{x} \\
\mathbf{y}
\end{array}\right]
$$

Here $a$ stands for a measure of anomalousness, since Eq. (9) is not a distance-based metric anymore. The HACD detector is designed to be more robust to image noise, blurring effects, and spectral differences ${ }^{11}$. All detectors in this paper except Euclidean distance are based on a global approach, by estimating sample statistics over the whole image instead of over a local region.

\subsection{Local co-registration adjustment}

To account for co-registration errors, and instead of improving georeferencing accuracy, changes can be considered in the area around a pixel ${ }^{12}$. For instance, by comparing the measurement vector $\mathbf{x}$ with the $3 \times 3$ area around the geospatial position of measurement vector $\mathbf{y}$, and considering the minimum distance as the best estimate of change. By doing this in the opposite direction as well, and consider the maximum of both minima as the best estimate, we get the following expression:

$$
d_{i j}=\max \left(\min \left(d\left(\mathbf{x}_{i j}, \mathbf{y}_{i j \in W}\right)\right), \min \left(d\left(\mathbf{x}_{i j \in W}, \mathbf{y}_{i j}\right)\right)\right)
$$


Here $W$ is the area around the geospatial position or pixel $(i, j)$ under test. In case of HACD detection, the distance $d$ in Eq. (10) can be replaced by the measure of anomalousness in Eq. (9).

\subsection{Object recognition}

Since operators who deal with target acquisition and situation awareness, are generally more interested in objects (e.g. vehicles, buildings) than in pixels, clustering is applied to those pixels that are spatially connected. Because at this stage we deal with binary detection masks, a simple region-growing spatial clustering scheme is used.

\subsection{False alarm reduction}

If the spatial resolution of the images is high enough, false alarm reduction based on spatial features is often applied. Many spatial features exist that define the size and shape of a target. Due to time constraints we were not able to fully investigate this topic, which is a field of research in itself. But to include some level of false alarm reduction in evaluating hyperspectral against broadband, we applied a filter on the target size in square meters (based on the number of pixels) as a single feature.

\subsection{Computation of ROC curves}

Comparison of the detected target objects and their ground-truth positions, and obtaining ROC curves, is based on association techniques that are common in for instance in target tracking ${ }^{13}$. Basis for association is their geospatial distance. The following procedure is applied:

1. Change detection (Section 3.1, 3.2)

2. Spatial clustering into target objects and optionally false alarm reduction (Section $3.3,3.4$ )

3. Compute centre-of-gravity of each object

4. Compute Euclidean distance between all centres-of-gravity and all ground-truth positions, resulting in a distance matrix

5. Sort distance matrix (smallest distance first)

6. Start association with smallest distance (an object can only once be associated to a ground-truth position)

7. Stop association if maximum distance is reached (maximum distance $=$ half size of the target + georeference error relative to ground truth), non-associated objects are considered as false alarms

8. Repeat this procedure with a different threshold to create ROC curve: fraction of associated objects (probability of detection) versus the number of non-associated objects per unit area (number of false alarms)

\section{RESULTS AND DISCUSSION}

Applying the abovementioned change detection strategies without false alarm reduction resulted in the ROC curves of Fig. 5. A local co-registration adjustement window of $5 \times 5$ pixels was applied, that is in line with the co-registration accuracy of both TASI and FLIR MWIR images. Maximum distance in association (Section 3.5, point 7) was chosen to be $2.5 \mathrm{~m}$, half the size of an average vehicle. Georeferencing error with respect to the ground truth is considered $0 \mathrm{~m}$ since the ground truth positions of the relocated vehicles were obtained from the images under test. In Fig. 5 we see that the TASI LWIR hyperspectral data in combination with HACD shows the best results. Applying false alarm reduction according to Section 3.4 resulted in the ROC curves of Fig. 6 . Here the threshold is set to $1.1 \mathrm{~m}^{2}$, that corresponds to three pixels in the TASI data, and to 18 pixels in the FLIR MWIR data. We see that the performance of FLIR MWIR significantly improves, opposite to TASI that does not change much. Still TASI in combination with HACD outperforms FLIR MWIR, but that is not true for the other detectors. In general, in LWIR and MWIR the contrast of disappeared vehicles (i.e. present in first image, not present in second image) is larger than that of appeared vehicles (i.e. not present in first image, present in second image) due to the thermal shadows that are left behind by the disappeared vehicles.

Reason why the variation in performance of TASI for the different detectors is larger than for FLIR MWIR is the higher contribution of thermal noise in TASI. Looking at the output of all detectors we see that Mahalanobis (with or without $\mathrm{CC}, \mathrm{CE}$ ) shows more noise than the output of HACD, see also Fig. 7. Apparently the distribution-based HACD detection 
strategy as described in Section 3.1 pays off for TASI. Similar observations are shown by Theiler ${ }^{11}$ in case of injecting noise in one of the original images. Note that $\mathrm{CC}$ further increases the noise at the output of the Mahalanobis detector which explains the worst ROC. In case of FLIR MWIR broadband, results illustrate that if the images contain less thermal noise, the results of the different change detection strategies are much closer. Here the simplest detector, Euclidean distance, shows an interesting alternative if no false alarm reduction is applied.
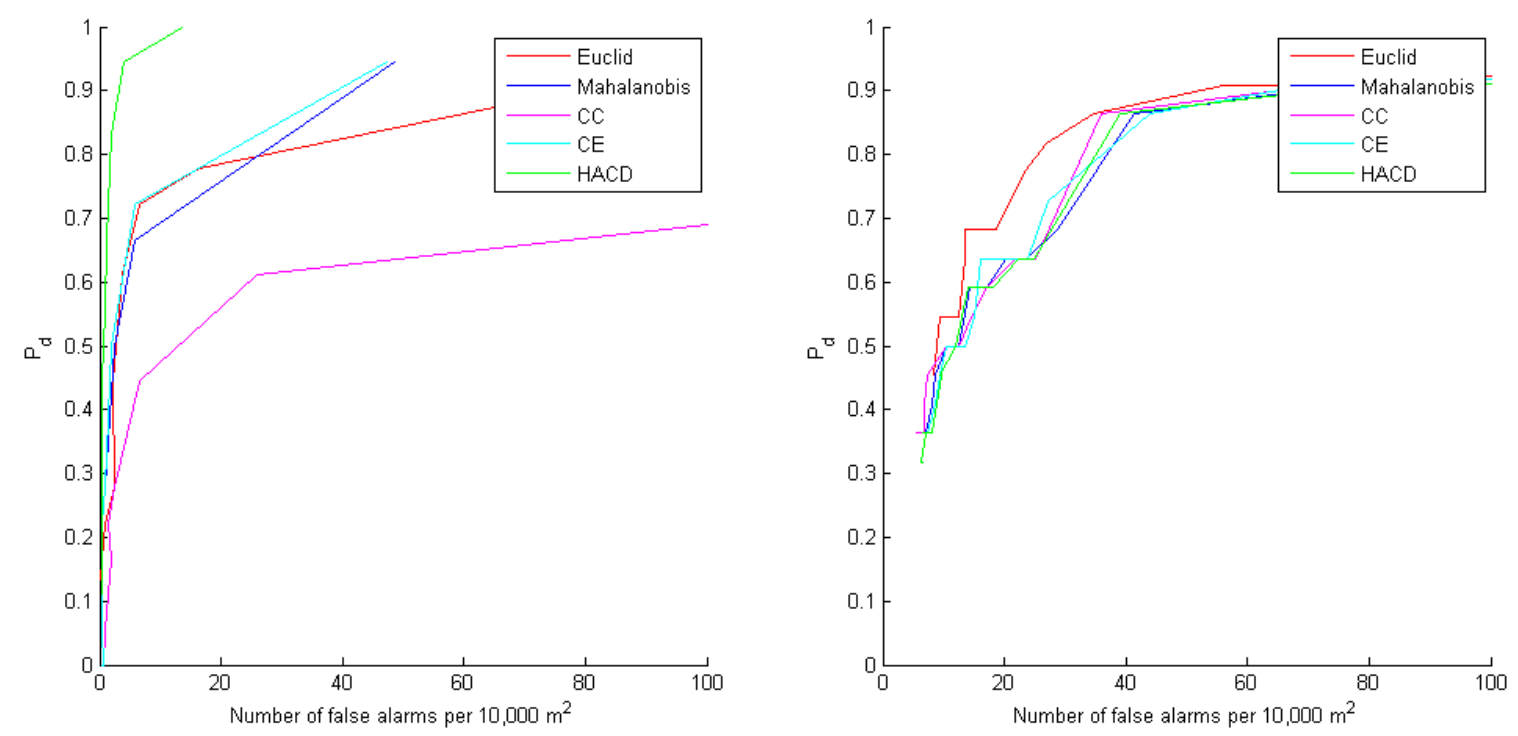

Figure 5. TASI LWIR (left) and FLIR MWIR (right) vehicle change detection ROC curves with local co-registration adjustement (no false alarm reduction). The unit area $10,000 \mathrm{~m}^{2}$ equals an area of $100 \mathrm{~m} \times 100 \mathrm{~m}$.
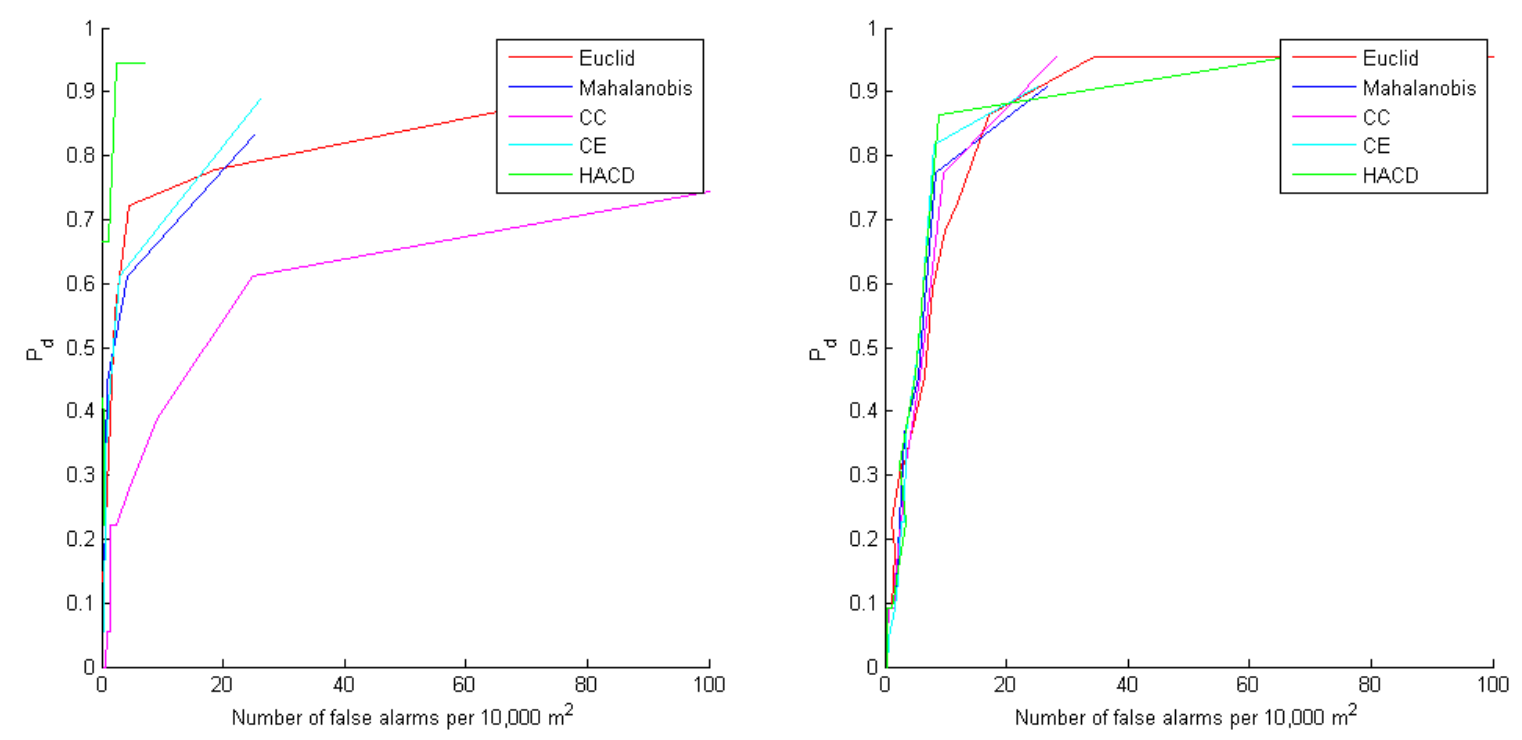

Figure 6. TASI LWIR (left) and FLIR MWIR (right) vehicle change detection ROC curves with local co-registration adjustement after rejecting all changes of $1.1 \mathrm{~m}^{2}$ and smaller. 

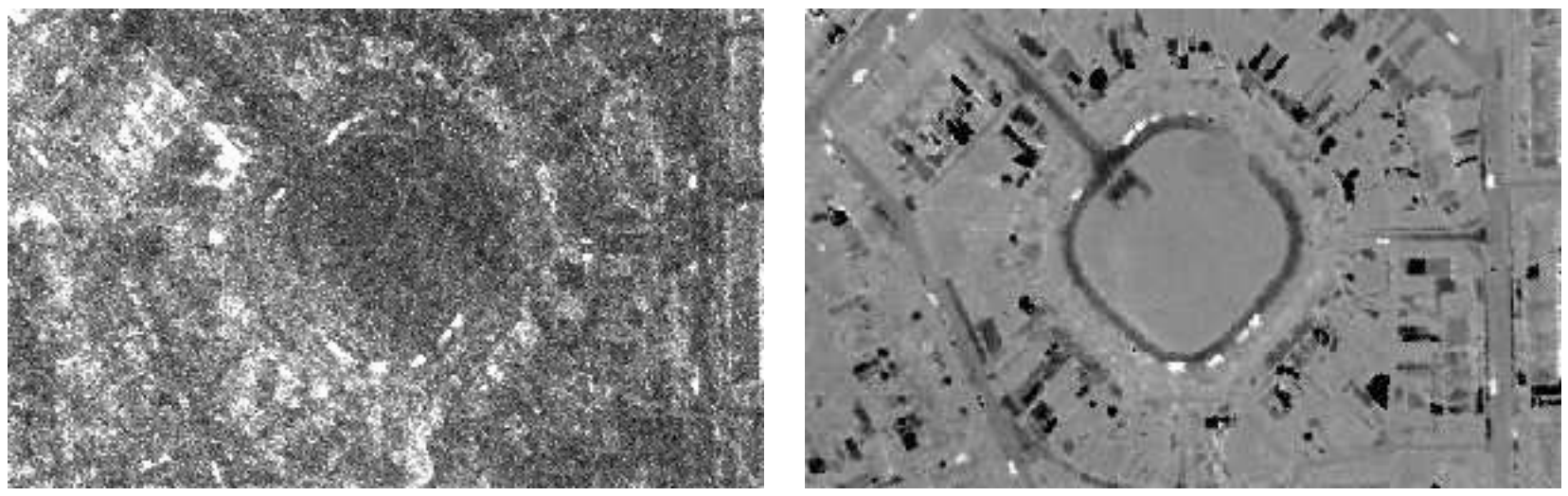

Figure 7. Mahalanobis distance of the residual vector (left) and HACD anomalousness (right) with local co-registration adjustment based on the TASI images of Fig. 1.
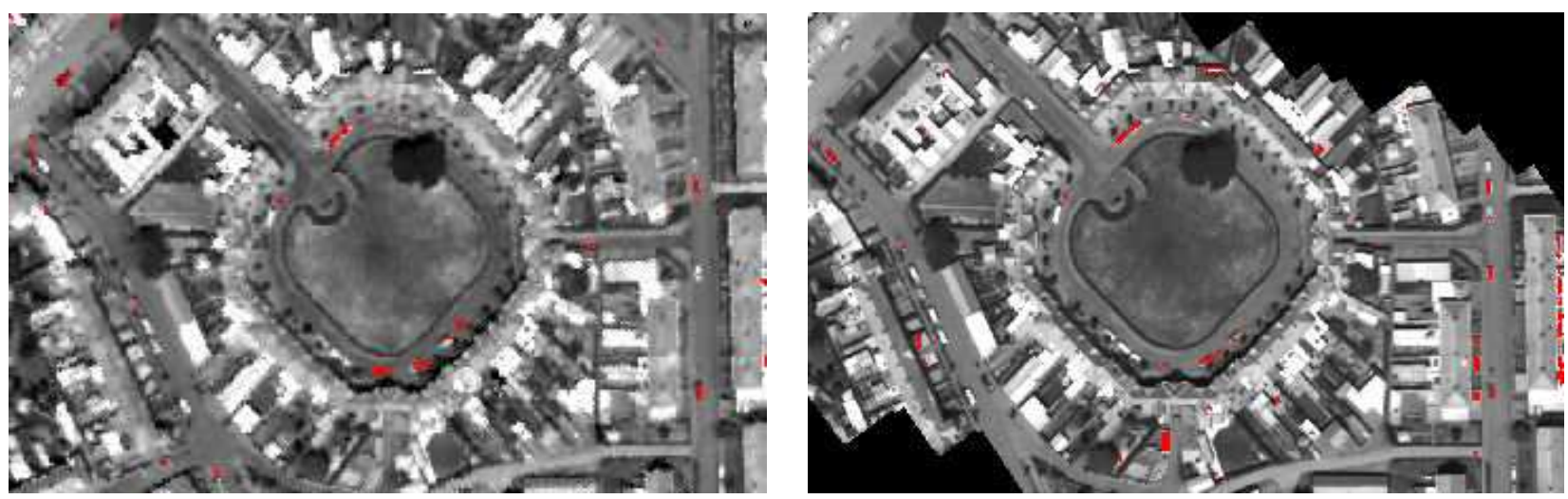

Figure 8. TASI LWIR (left) and FLIR MWIR (right) vehicle change detection after false alarm reduction corresponding with the optimum detector (HACD) and thresholds of Fig. 6.

In the analysis so far we considered the small differences in time interval, area of interest, and the differences in wavelength negligible. Coming back to Fig. 6, and to see how the detection results look like for optimum detectors and threshold, Fig. 8 shows examples of TASI and FLIR MWIR. Here we see that in case of FLIR MWIR false alarms are more often caused by buildings (roofs) and their shadows, which suggests that the MWIR channels contain a higher contribution of reflection in comparison with emission. Something that seems to be confirmed by the images in Fig. 3 , where the radiance of $3.9 \mu \mathrm{m}$ is higher than of $4.8 \mu \mathrm{m}$ for certain objects (again mainly roofs) given their reddish colour. This put forward the idea to compare TASI and FLIR MWIR assuming the same image characteristics (number of bands, bandwidth, spatial resolution) and see what the difference between LWIR and MWIR actually is. Therefore TASI was spectrally resampled to two bands (respectively $8.3 \mu \mathrm{m}$ and $9.2 \mu \mathrm{m}$ ) by averaging two times six bands to a bandwidth of roughly $600 \mathrm{~nm}$. FLIR MWIR was spatially resampled to a ground resolution of $0.60 \mathrm{~m}$. Change detection strategies without false alarm reduction applied to these results are shown in Fig. 9. Here we see that LWIR has a clear advantage over MWIR, and that we can not neglect the differences in wavelength.

Comparing Fig. 5 with Fig. 9 it is interesting to see that it pays to apply HACD to 32 LWIR bands (i.e. TASI) instead of 2 broader LWIR bands. Looking at the other detectors it is clear that the thermal noise has reduced. However, what we can not evaluate is the performance of LWIR hyperspectral data compared to LWIR high spatial-resolution data. In case of FLIR MWIR, a higher spatial resolution in combination with false alarm reduction yields the best ROC performance. The somewhat irregular shapes of the ROC curves are caused by the object-level evaluation: at certain thresholds objects will be combined causing the probability of detection or the number of false alarms to drop. 

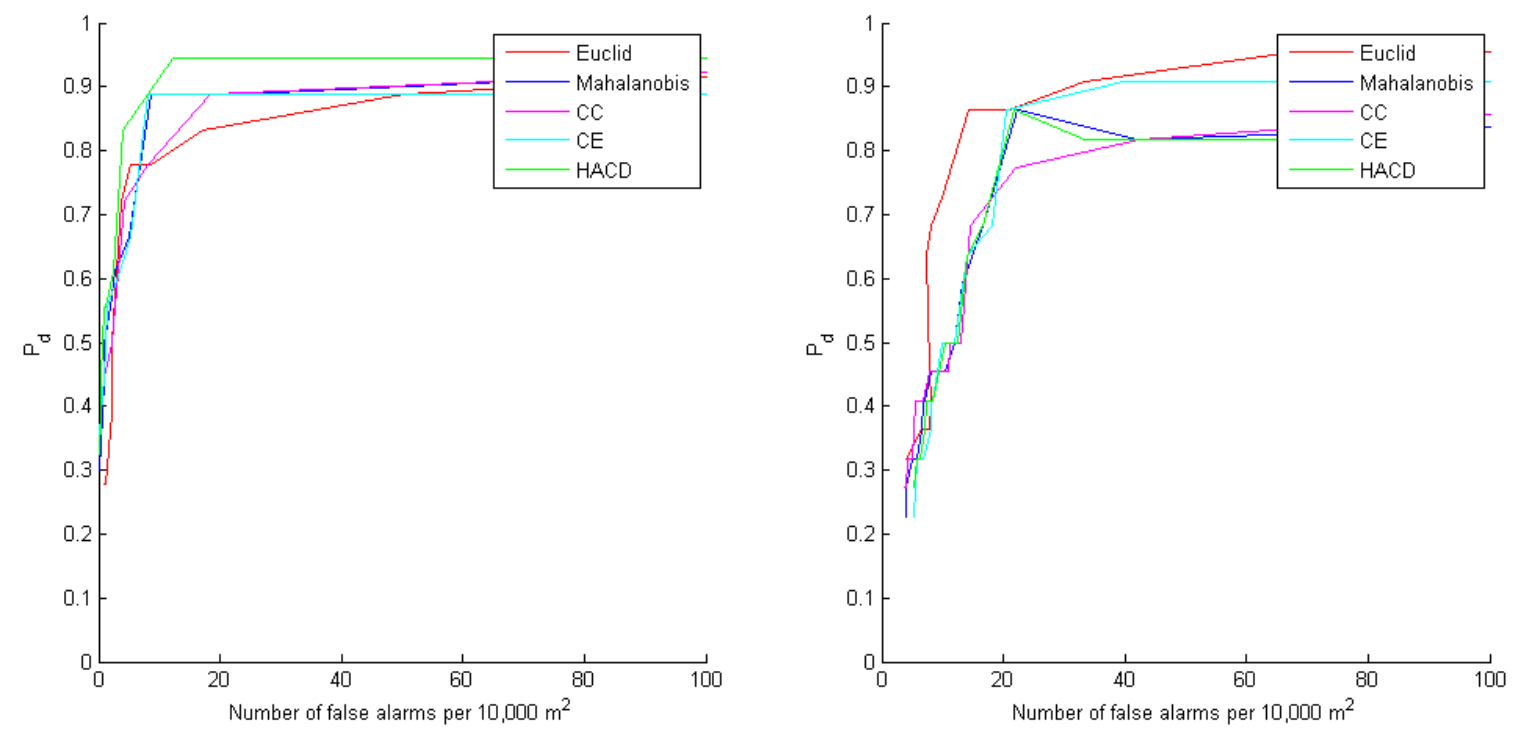

Figure 9. TASI LWIR (left) and FLIR MWIR (right) vehicle change detection ROC curves with local co-registration adjustement after resampling to two bands with a spectral width of $600 \mathrm{~nm}$ (FWHM) and a spatial resolution of $0.60 \mathrm{~m}$ (no false alarm reduction). Note the drop in probability of detection of FLIR MWIR for Mahalanobis and HACD that is due to spatial clustering of two target objects into one object.

\section{CONCLUSIONS AND RECOMMENDATIONS}

Based on the results obtained in this study we conclude that in target detection and situation awareness of urban areas, LWIR hyperspectral has an advantage over MWIR broadband change detection if a strategy is chosen that is robust to the thermal noise in the hyperspectral imagery. In this study that strategy is Hyperbolic Anomalous Change Detection",

${ }^{11}$. MWIR high spatial-resolution broadband results show that it helps to apply a false alarm reduction strategy based on spatial processing. In general, in LWIR and MWIR the contrast of disappeared vehicles (i.e. present in first image, not present in second image) is larger than that of appeared vehicles (i.e. not present in first image, present in second image) due to the thermal shadows that are left behind by the disappeared vehicles.

To support these conclusions it will be interesting to investigate other atmospheric conditions, nighttime imagery, and other urban environments. With that are topics such as the effects of atmospheric corrections, Temperature-Emissivity Separation (TES), and local detection strategies that estimate sample statistics over local regions instead of the whole image $^{10}$. To improve the change detection performance of high spatial-resolution broadband sensors, a more elaborate false alarm reduction strategy will likely pay off. In a new experiment it is recommended to also include LWIR high spatial-resolution sensors, next to LWIR hyperspectral sensors, for comparison.

\section{REFERENCES}

[1] Renhorn, I., Axelsson, M., Benoist, K., Borghys, D., Boucher, Y., Briottet, X., De Ceglie, S., Dekker, R., Dimmeler, A., Dost, R., Friman, O., Kåsen, I., Maerker, J., van Persie, M., Resta, S., Schwering, P., Shimoni, M. and Haavardsholm, T.V., "Detection in Urban scenario using Combined Airborne imaging Sensors," Proc. SPIE 8353 (2013).

[2] “TASI-600 Airborne Hyperspectral Thermal Imager," ITRES Research Ltd., 2010, <http://www.itres.com/products/imagers/tasi600/> (5 April 2013). 
[3] "FLIR SC7000 Series," FLIR Systems, Inc., 2011, <http://www.flir.com/cs/emea/en/view/?id=42576> (5 April 2013).

[4] Manolakis, D. and Shaw, G., "Detection Algorithms for Hyperspectral Imaging Applications," IEEE Signal Processing Magazine, pp. 12-16 (January 2002).

[5] Pignatti, S., Lapenna, V., Palombo, A., Pascucci, S., Pergola, N. and Cuomo, V., "An advanced tool of the CNR IMAA EO facilities: Overview of the TASI-600 hyperspectral thermal spectrometer," Proc. ${ }^{\text {rd }}$ Workshop on Hyperspectral Image and Signal Processing (WHISPERS), pp. 1-4 (2011).

[6] Schaum, A. and Stocker, A., "Subclutter target detection using sequences of thermal infrared multispectral imagery," Proc. SPIE 3071, pp. 12-22 (1997).

[7] Stein, D.W.J., Beaven, S.G., Hoff, L.E. Winter, E.M., Schaum, A.P. and Stocker A.D., “Anomaly Detection from Hyperspectral Imagery,” IEEE Signal Processing Magazine, pp. 58-69 (January 2002).

[8] Schaum, A. and Stocker, A., "Hyperspectral change detection and supervised matched filtering based on Covariance Equalization," Proc. SPIE 5425, pp. 77-90 (2004).

[9] Theiler, J. and Perkins, S., "Proposed Framework for Anomalous Change Detection," Proc. ICML Workshop on Machine Learning Algorithms for Surveillance and Event Detection, pp. 7-14 (2006).

[10] Eismann, M.T., Meola, J. and Hardie, R.C., "Hyperspectral Change Detection in the Presence of Diurnal and Seasonal Variations," IEEE Trans. Geoscience And Remote Sensing 46(1), pp. 237-249 (2008).

[11] Theiler, J., "Quantitative comparison of quadratic covariance-based anomalous change detectors," Applied Optics 47(28), pp. F12-F26 (2008).

[12] Theiler, J. and Wohlberg, B., "Local Coregistration Adjustment for Anomalous Change Detection," IEEE Trans. Geoscience And Remote Sensing 50(8), pp. 3107-3116 (2012).

[13] Blackman, S. and Popoli, R., [Design and Analysis of Modern Tracking Systems], Artech House, Norwood, MA (1999). 